1 Hacettepe Journal of Mathematics and Statistics

$\bigcap$ Volume 45 (5) (2016), $1441-1448$

\title{
On the spectral norms of some special $g$-circulant matrices
}

\author{
Shou-Qiang Shen ${ }^{* \dagger}$, Wei-Jun Liu ${ }^{\ddagger}$ and Jun-Jie $\mathrm{He}^{\S}$
}

\begin{abstract}
In the present paper, we give upper and lower bounds for the spectral norm of $g$-circulant matrix, whose the first row entries are the classical Horadam numbers $U_{i}^{(a, b)}$. In addition, we also establish an explicit formula of the spectral norm for $g$-circulant matrix with the first row $\left(\left[U_{0}^{(a, b)}\right]^{2},\left[U_{1}^{(a, b)}\right]^{2}, \cdots,\left[U_{n-1}^{(a, b)}\right]^{2}\right)$.
\end{abstract}

Keywords: $\quad g$-Circulant matrix; Spectral norm; Horadam number; Fibonacci number; Lucas number

2000 AMS Classification: 15A45, 15A60.

Received : 08.01.2015 Accepted: 01.03.2016 Doi : 10.15672/HJMS.20164515690

\section{Introduction and Preliminaries}

Many generalizations of the Fibonacci and Lucas sequences have been introduced and studied [1-4]. Here we use the classical Horadam sequence $\left\{U_{n}^{(a, b)}\right\}_{n \in N}$, which is defined in [4]:

$$
U_{n}^{(a, b)}=A U_{n-1}^{(a, b)}+B U_{n-2}^{(a, b)}, \quad U_{0}^{(a, b)}=a, \quad U_{1}^{(a, b)}=b,
$$

where $a, b \in R$ and $A^{2}+4 B>0$. Obviously, if we choose $A=B=1$ in (1), then the generalized Fibonacci sequence $\left\{F_{n}^{(a, b)}\right\}_{n \in N}$ is obtained. Further more, when $a=0, b=1$ and $a=2, b=1$, the sequence $\left\{F_{n}^{(a, b)}\right\}_{n \in N}$ reduces to the well-known Fibonacci sequence $\left\{F_{n}\right\}_{n \in N}$ and Lucas sequence $\left\{L_{n}\right\}_{n \in N}$, respectively.

For the Horadam sequence $\left\{U_{n}^{(a, b)}\right\}_{n \in N}$, the following generalization of the Binet's formula of Fibonacci number holds [4]:

$$
U_{n}^{(a, b)}=c_{1} \alpha^{n}+c_{2} \beta^{n},
$$

*Supported by the Key Research Project of Higher school in Henan Province(No. 15A110040).

†School of Mathematics and Statistics, Central South University, 410083 Changsha, China. Corresponding author,

Email : shenshouqiang@126.com

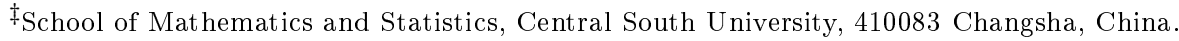

$\S$ Department of Mathematics, Xinyang Normal University, 464000 Xinyang, China. 
where

$$
\begin{aligned}
& c_{1}=\frac{a\left(A^{2}+4 B\right)+(2 b-a A) \sqrt{A^{2}+4 B}}{2\left(A^{2}+4 B\right)}, \\
& c_{2}=\frac{a\left(A^{2}+4 B\right)-(2 b-a A) \sqrt{A^{2}+4 B}}{2\left(A^{2}+4 B\right)}, \\
& \alpha=\frac{A+\sqrt{A^{2}+4 B}}{2}, \beta=\frac{A-\sqrt{A^{2}+4 B}}{2} .
\end{aligned}
$$

Recently, there has been much interest in investigation of some special matrices. Akbulak and Bozkurt [5] found the lower and upper bounds for the spectral norms of Toeplitz matrices $\mathcal{A}=\left[F_{i-j}\right]_{i, j=1}^{n}$ and $\mathcal{B}=\left[L_{i-j}\right]_{i, j=1}^{n}$, then Shen [6] generalized these results. Solak $[7,8]$ gave the upper and lower bounds for the spectral norms of circulant matrices whose entries are Fibonacci and Lucas numbers. Then İpek [9] investigated an improved estimation for the spectral norms of these matrices. In addition, there have been several articles focus on the spectral distribution and norms of $g$-circulant matrices. Bose et al. [10] listed the limiting spectral distribution for a class of $g$-circulant matrices with heavy tailed input sequence. Zhou and Jiang [11] derived some explicit formulas for the spectral norms of $g$-circulant matrices whose the first row entries are Fibonacci number, Lucas number and their powers.

Besides, Shen et al. [12] gave some feasible computational formulas for the determinants and inverses of the circulant matrices $\mathcal{A}_{n}=\operatorname{Circ}\left(F_{1}, F_{2}, \cdots, F_{n}\right)$ and $\mathcal{B}_{n}=\operatorname{Circ}\left(L_{1}\right.$, $\left.L_{2}, \cdots, L_{n}\right)$, then Yazlik and Taskara [13] generalized all results from [12]. Stanimirović et al. [4] defined an $n \times n$ Toeplitz matrix $\mathcal{U}_{n}^{(a, b, s)}=\left[u_{i, j}^{(a, b, s)}\right](i, j=1,2, \cdots, n)$ of type $s$, where

$$
u_{i, j}^{(a, b, s)}= \begin{cases}U_{i-j+1}^{(a, b)}, & i-j+s \geq 0, \\ 0, & i-j+s<0 .\end{cases}
$$

then the inverse of the matrix $\mathcal{U}_{n}^{(a, b, 0)}$ was derived, and correlations between the matrix $\mathcal{U}_{n}^{(a, b, 0)}$ and the generalized Pascal matrices of the first and the second kinds were considered. In addition, Shen and He [14] also established an explicit formula of the Moore-Penrose inverse for the matrix $\mathcal{U}_{n}^{(a, b,-1)}$.

In this paper, let $\mathcal{A}_{U}$ and $\mathcal{A}_{U^{2}}$ be two $g$-circulant matrices, whose the first row entries are $\left(U_{0}^{(a, b)}, U_{1}^{(a, b)}, \cdots, U_{n-1}^{(a, b)}\right)$ and $\left(\left[U_{0}^{(a, b)}\right]^{2},\left[U_{1}^{(a, b)}\right]^{2}, \cdots,\left[U_{n-1}^{(a, b)}\right]^{2}\right)$, respectively. We give upper and lower bounds for the spectral norm of matrix $\mathcal{A}_{U}$, and establish an explicit formula of the spectral norm for matrix $\mathcal{A}_{U^{2}}$, then generalize the main results in [11].

Now we give some preliminaries related to our study. A matrix $\mathcal{A} \in M_{n}$ is called a $g$-circulant matrix if it is of the form

$$
\mathcal{A}=\left(\begin{array}{ccccc}
a_{0} & a_{1} & a_{2} & \cdots & a_{n-1} \\
a_{n-g} & a_{n-g+1} & a_{n-g+2} & \cdots & a_{n-g-1} \\
a_{n-2 g} & a_{n-2 g+1} & a_{n-2 g+2} & \cdots & a_{n-2 g-1} \\
\vdots & \vdots & \vdots & \ddots & \vdots \\
a_{g} & a_{g+1} & a_{g+2} & \cdots & a_{g-1}
\end{array}\right)
$$

where $g$ is a nonnegative integer and each of the subscripts is understood to be reduced modulo $n$. Obviously, when $g=1$ or $g=n+1$, the $g$-circulant matrix $\mathcal{A}$ reduces to the standard circulant matrix. 
For any $\mathcal{A}=\left[a_{i j}\right] \in M_{m, n}$. The well-known Frobenius (or Euclidean) norm of matrix $\mathcal{A}$ is

$$
\|\mathcal{A}\|_{F}=\left[\sum_{i=1}^{m} \sum_{j=1}^{n}\left|a_{i j}\right|^{2}\right]^{\frac{1}{2}}
$$

and also the spectral norm of matrix $\mathcal{A}$ is

$$
\|\mathcal{A}\|_{2}=\sqrt{\max _{1 \leq i \leq n} \lambda_{i}\left(\mathcal{A}^{H} \mathcal{A}\right)}
$$

where $\lambda_{i}\left(\mathcal{A}^{H} \mathcal{A}\right)$ is eigenvalue of $\mathcal{A}^{H} \mathcal{A}$ and $\mathcal{A}^{H}$ is conjugate transpose of matrix $\mathcal{A}$. Then the following inequality holds:

$$
\frac{1}{\sqrt{n}}\|\mathcal{A}\|_{F} \leq\|\mathcal{A}\|_{2} \leq\|\mathcal{A}\|_{F}
$$

Lemma $1^{[15]}$ An $n \times n$ matrix $Q_{g}$ is unitary if and only if $(n, g)=1$, where $Q_{g}$ is a g-circulant matrix with the first row $(1,0, \cdots, 0)$.

Lemma $2^{[15]} \quad \mathcal{A}$ is a g-circulant matrix with the first row $\left(a_{0}, a_{1}, \cdots, a_{n-1}\right)$ if and only if $\mathcal{A}=Q_{g} C$, where $C$ is a circulant matrix with the first row $\left(a_{0}, a_{1}, \cdots, a_{n-1}\right)$.

Lemma $3^{[16]} \quad$ Let $\mathcal{A}=\left[a_{i j}\right] \in M_{n}$ is a nonnegative matrix, Then its spectral radius $\rho(\mathcal{A})$ satisfies the following inequality

$$
\min _{1 \leq i \leq n} \sum_{j=1}^{n} a_{i j} \leq \rho(\mathcal{A}) \leq \max _{1 \leq i \leq n} \sum_{j=1}^{n} a_{i j} .
$$

Lemma 4 For the Horadam sequence $\left\{U_{n}^{(a, b)}\right\}_{n \in N}$ satisfying $B \neq-1$ and $B \pm A \neq 1$, the following identity is valid:

$$
\sum_{i=0}^{n-1}\left[U_{i}^{(a, b)}\right]^{2}=\frac{M-\left[U_{n}^{(a, b)}\right]^{2}+B^{2}\left[U_{n-1}^{(a, b)}\right]^{2}}{(1-B)^{2}-A^{2}}+\frac{2\left(a^{2} B+a b A-b^{2}\right)\left[1-(-B)^{n}\right]}{(1+B)\left(A^{2}+4 B\right)}
$$

where $M=a^{2}-(a A-b)^{2}-\frac{2(1+B)\left(a^{2} B+a b A-b^{2}\right)\left[1-(-B)^{n}\right]}{A^{2}+4 B}$.

Proof: From $B \pm A \neq 1$, we get $\alpha \neq \pm 1$ and $\beta \neq \pm 1$, applying identities $U_{n}^{(a, b)}=$ $c_{1} \alpha^{n}+c_{2} \beta^{n}$ and $\alpha \beta=-B$, then the following is valid

$$
\begin{aligned}
\sum_{i=0}^{n-1}\left[U_{i}^{(a, b)}\right]^{2}= & \sum_{i=0}^{n-1}\left(c_{1} \alpha^{i}+c_{2} \beta^{i}\right)^{2}=c_{1}^{2} \sum_{i=0}^{n-1} \alpha^{2 i}+c_{2}^{2} \sum_{i=0}^{n-1} \beta^{2 i}+2 c_{1} c_{2} \sum_{i=0}^{n-1}(\alpha \beta)^{i} \\
= & c_{1}^{2} \cdot \frac{1-\alpha^{2 n}}{1-\alpha^{2}}+c_{2}^{2} \cdot \frac{1-\beta^{2 n}}{1-\beta^{2}}+2 c_{1} c_{2} \cdot \frac{1-(\alpha \beta)^{n}}{1-\alpha \beta} \\
= & \frac{c_{1}^{2}+c_{2}^{2}-\left(c_{2}^{2} \alpha^{2}+c_{1}^{2} \beta^{2}\right)-\left(c_{1}^{2} \alpha^{2 n}+c_{2}^{2} \beta^{2 n}\right)+(\alpha \beta)^{2}\left(c_{1}^{2} \alpha^{2 n-2}+c_{2}^{2} \beta^{2 n-2}\right)}{\left(1-\alpha^{2}\right)\left(1-\beta^{2}\right)} \\
& +2 c_{1} c_{2} \frac{1-(-B)^{n}}{1+B} .
\end{aligned}
$$

By using identities $\alpha+\beta=A$ and $\alpha-\beta=\sqrt{A^{2}+4 B}$, we have

$$
\begin{aligned}
& c_{1}=\frac{a}{2}+\frac{2 b-a A}{2 \sqrt{A^{2}+4 B}}=\frac{a}{2}+\frac{2 b-a(\alpha+\beta)}{2(\alpha-\beta)}=\frac{b-a \beta}{\alpha-\beta}, \\
& c_{2}=\frac{a}{2}-\frac{2 b-a A}{2 \sqrt{A^{2}+4 B}}=\frac{a}{2}-\frac{2 b-a(\alpha+\beta)}{2(\alpha-\beta)}=\frac{a \alpha-b}{\alpha-\beta} .
\end{aligned}
$$


So we obtain

$$
\begin{aligned}
c_{2}^{2} \alpha^{2}+c_{1}^{2} \beta^{2} & =\left(c_{2} \alpha+c_{1} \beta\right)^{2}-2 c_{1} c_{2} \alpha \beta=\left(\frac{a \alpha-b}{\alpha-\beta} \cdot \alpha+\frac{b-a \beta}{\alpha-\beta} \cdot \beta\right)^{2}-2 c_{1} c_{2} \alpha \beta \\
& =[a(\alpha+\beta)-b]^{2}-2 c_{1} c_{2} \alpha \beta=(a A-b)^{2}+2 c_{1} c_{2} B .
\end{aligned}
$$

Since $c_{1}^{2}+c_{2}^{2}=a^{2}-2 c_{1} c_{2}$ and

$$
\begin{gathered}
c_{1}^{2} \alpha^{2 n}+c_{2}^{2} \beta^{2 n}=\left(c_{1} \alpha^{n}+c_{2} \beta^{n}\right)^{2}-2 c_{1} c_{2}(\alpha \beta)^{n}=\left[U_{n}^{(a, b)}\right]^{2}-2 c_{1} c_{2}(-B)^{n}, \\
(\alpha \beta)^{2}\left(c_{1}^{2} \alpha^{2 n-2}+c_{2}^{2} \beta^{2 n-2}\right)=B^{2}\left[U_{n-1}^{(a, b)}\right]^{2}-2 c_{1} c_{2}(-B)^{n+1} .
\end{gathered}
$$

While $c_{1} c_{2}=\frac{a^{2} B+a b A-b^{2}}{A^{2}+4 B}$, hence

$$
\begin{aligned}
\sum_{i=0}^{n-1}\left[U_{i}^{(a, b)}\right]^{2}= & \frac{a^{2}-(a A-b)^{2}-2 c_{1} c_{2}(1+B)\left[1-(-B)^{n}\right]-\left[U_{n}^{(a, b)}\right]^{2}+B^{2}\left[U_{n-1}^{(a, b)}\right]^{2}}{(1+\alpha \beta)^{2}-(\alpha+\beta)^{2}} \\
& +2 c_{1} c_{2} \frac{1-(-B)^{n}}{1+B} \\
= & \frac{a^{2}-(a A-b)^{2}-\frac{2(1+B)\left(a^{2} B+a b A-b^{2}\right)\left[1-(-B)^{n}\right]}{A^{2}+4 B}-\left[U_{n}^{(a, b)}\right]^{2}+B^{2}\left[U_{n-1}^{(a, b)}\right]^{2}}{(1-B)^{2}-A^{2}} \\
& +\frac{2\left(a^{2} B+a b A-b^{2}\right)\left[1-(-B)^{n}\right]}{(1+B)\left(A^{2}+4 B\right)} .
\end{aligned}
$$

Thus the proof is completed.

\section{Main Results}

Theorem 1 Let $\mathcal{A}_{U}$ be as the matrix in (1.6), with $a_{i}=U_{i}^{(a, b)}(i=0,1, \cdots, n-1)$ in the first row of $\mathcal{A}_{U}$. If $B \neq-1, B \pm A \neq 1$ and $(n, g)=1$, then we have

$$
\begin{gathered}
\begin{array}{c}
\sqrt{\frac{M-\left[U_{n}^{(a, b)}\right]^{2}+B^{2}\left[U_{n-1}^{(a, b)}\right]^{2}}{(1-B)^{2}-A^{2}}+}+\frac{2\left(a^{2} B+a b A-b^{2}\right)\left[1-(-B)^{n}\right]}{(1+B)\left(A^{2}+4 B\right)} \leq\left\|\mathcal{A}_{U}\right\|_{2} \\
\quad \leq \frac{1}{\sqrt{A^{2}+4 B}}\left[\frac{|b-a \beta|\left(1-|\alpha|^{n}\right)}{1-|\alpha|}+\frac{|b-a \alpha|\left(1-|\beta|^{n}\right)}{1-|\beta|}\right], \\
\text { where } \alpha=\frac{A+\sqrt{A^{2}+4 B}}{2}, \beta=\frac{A-\sqrt{A^{2}+4 B}}{2} \text { and } M=a^{2}-(a A-b)^{2}-\frac{2(1+B)\left(a^{2} B+a b A-b^{2}\right)\left[1-(-B)^{n}\right]}{A^{2}+4 B} .
\end{array}
\end{gathered}
$$

Proof: Applying the definition of Frobenius norm and formula (1.9), we have

$$
\begin{aligned}
\left\|\mathcal{A}_{U}\right\|_{F}^{2} & =n \sum_{i=0}^{n-1}\left[U_{i}^{(a, b)}\right]^{2} \\
& =n\left(\frac{M-\left[U_{n}^{(a, b)}\right]^{2}+B^{2}\left[U_{n-1}^{(a, b)}\right]^{2}}{(1-B)^{2}-A^{2}}+\frac{2\left(a^{2} B+a b A-b^{2}\right)\left[1-(-B)^{n}\right]}{(1+B)\left(A^{2}+4 B\right)}\right),
\end{aligned}
$$

where $M=a^{2}-(a A-b)^{2}-\frac{2(1+B)\left(a^{2} B+a b A-b^{2}\right)\left[1-(-B)^{n}\right]}{A^{2}+4 B}$. Hence from (1.7), we obtain

$$
\begin{aligned}
\left\|\mathcal{A}_{U}\right\|_{2} & \geq \frac{1}{\sqrt{n}}\left\|\mathcal{A}_{U}\right\|_{F} \\
& =\sqrt{\frac{M-\left[U_{n}^{(a, b)}\right]^{2}+B^{2}\left[U_{n-1}^{(a, b)}\right]^{2}}{(1-B)^{2}-A^{2}}+\frac{2\left(a^{2} B+a b A-b^{2}\right)\left[1-(-B)^{n}\right]}{(1+B)\left(A^{2}+4 B\right)}}
\end{aligned}
$$

On the other hand, using the results from Lemma 1 and Lemma 2, one can verify

$$
\left(\mathcal{A}_{U}\right)^{H} \mathcal{A}_{U}=\left(Q_{g} C\right)^{H} Q_{g} C=C^{H}\left(Q_{g}\right)^{H} Q_{g} C=C^{H} I_{n} C=C^{H} C,
$$


where $C$ is a circulant matrix with the first row $\left(U_{0}^{(a, b)}, U_{1}^{(a, b)}, \cdots, U_{n-1}^{(a, b)}\right)$ and $I_{n}$ is an identity matrix. Hence the spectral norm of matrix $\mathcal{A}_{U}$ is the same as that of $C$. Let $f(x)=\sum_{i=0}^{n-1} U_{i}^{(a, b)} x^{i}$ be a scalar-valued polynomial, and $\pi_{n}$ be an $n \times n$ circulant matrix with the first row $(0,1, \cdots, 0)$, then we get

$$
C=f\left(\pi_{n}\right)=\sum_{i=0}^{n-1} U_{i}^{(a, b)} \pi_{n}^{i}
$$

hence

$$
\left\|\mathcal{A}_{U}\right\|_{2}=\|C\|_{2}=\left\|\sum_{i=0}^{n-1} U_{i}^{(a, b)} \pi_{n}^{i}\right\|_{2} \leq \sum_{i=0}^{n-1}\left\|U_{i}^{(a, b)} \pi_{n}^{i}\right\|_{2} \leq \sum_{i=0}^{n-1}\left|U_{i}^{(a, b)}\right|\left\|\pi_{n}\right\|_{2}^{i} .
$$

Since $\pi_{n}^{H} \pi_{n}=I_{n}$, then we have

$$
\left\|\pi_{n}\right\|_{2}=\sqrt{\max _{1 \leq i \leq n} \lambda_{i}\left(\pi_{n}^{H} \pi_{n}\right)}=1 .
$$

Note that $|\alpha| \neq 1$ and $|\beta| \neq 1$, hence we obtain

$$
\begin{aligned}
\left\|\mathcal{A}_{U}\right\|_{2} & \leq \sum_{i=0}^{n-1}\left|U_{i}^{(a, b)}\right|=\sum_{i=0}^{n-1}\left|c_{1} \alpha^{i}+c_{2} \beta^{i}\right| \leq\left|c_{1}\right| \sum_{i=0}^{n-1}|\alpha|^{i}+\left|c_{2}\right| \sum_{i=0}^{n-1}|\beta|^{i} \\
& =\left|c_{1}\right| \frac{1-|\alpha|^{n}}{1-|\alpha|}+\left|c_{2}\right| \frac{1-|\beta|^{n}}{1-|\beta|}=\frac{1}{\alpha-\beta}\left[\frac{|b-a \beta|\left(1-|\alpha|^{n}\right)}{1-|\alpha|}+\frac{|b-a \alpha|\left(1-|\beta|^{n}\right)}{1-|\beta|}\right] \\
& =\frac{1}{\sqrt{A^{2}+4 B}}\left[\frac{|b-a \beta|\left(1-|\alpha|^{n}\right)}{1-|\alpha|}+\frac{|b-a \alpha|\left(1-|\beta|^{n}\right)}{1-|\beta|}\right],
\end{aligned}
$$

where $\alpha=\frac{A+\sqrt{A^{2}+4 B}}{2}, \beta=\frac{A-\sqrt{A^{2}+4 B}}{2}$. Thus the proof is completed.

Example Let $\mathcal{A}_{F}$ be a 4-circulant matrix of the order 5 with the first row $\left(F_{0}^{(0,-1)}, F_{1}^{(0,-1)}\right.$, $\left.\cdots, F_{4}^{(0,-1)}\right)$, then

$$
\sqrt{15} \leq\left\|\mathcal{A}_{F}\right\|_{2} \leq 3+\frac{12}{\sqrt{5}}
$$

Theorem 2 Let $\mathcal{A}_{U^{2}}$ be as (1.6), with $a_{i}=\left[U_{i}^{(a, b)}\right]^{2}(i=0,1, \cdots, n-1)$ in the first row of $\mathcal{A}_{U^{2}}$. If $B \neq-1, B \pm A \neq 1$ and $(n, g)=1$, then we have the following identity

$$
\left\|\mathcal{A}_{U^{2}}\right\|_{2}=\frac{M-\left[U_{n}^{(a, b)}\right]^{2}+B^{2}\left[U_{n-1}^{(a, b)}\right]^{2}}{(1-B)^{2}-A^{2}}+\frac{2\left(a^{2} B+a b A-b^{2}\right)\left[1-(-B)^{n}\right]}{(1+B)\left(A^{2}+4 B\right)},
$$

where $M=a^{2}-(a A-b)^{2}-\frac{2(1+B)\left(a^{2} B+a b A-b^{2}\right)\left[1-(-B)^{n}\right]}{A^{2}+4 B}$.

Proof: Applying the results from Lemma 1 and Lemma 2, the following is valid

$$
\left(\mathcal{A}_{U^{2}}\right)^{H} \mathcal{A}_{U^{2}}=\left(Q_{g} C\right)^{H} Q_{g} C=C^{H}\left(Q_{g}\right)^{H} Q_{g} C=C^{H} I_{n} C=C^{H} C,
$$

where $C=\left[c_{i j}\right] \in M_{n}$ is a circulant matrix with the first row $\left(\left[U_{0}^{(a, b)}\right]^{2},\left[U_{1}^{(a, b)}\right]^{2}, \ldots,\left[U_{n-1}^{(a, b)}\right]^{2}\right)$. Hence the spectral norm of matrix $\mathcal{A}_{U^{2}}$ is the same as that of $C$.

Since the circulant matrix $C$ is normal, there exists a unitary matrix $V \in M_{n}$ such that $V^{H} C V=\operatorname{diag}\left(\lambda_{1}, \lambda_{2}, \cdots, \lambda_{n}\right)$, where $\lambda_{i}$ is eigenvalue of $C$, hence

$$
V^{H} C^{H} C V=\operatorname{diag}\left(\left|\lambda_{1}\right|^{2},\left|\lambda_{2}\right|^{2}, \cdots,\left|\lambda_{n}\right|^{2}\right) .
$$

Thus, the spectral norm of $C$ is given by its spectral radius. Also since $C$ is nonnegative, its spectral radius $\rho(C)$ satisfies the following inequality:

$$
\min _{1 \leq i \leq n} \sum_{j=1}^{n} c_{i j} \leq \rho(C) \leq \max _{1 \leq i \leq n} \sum_{j=1}^{n} c_{i j}
$$


While

$$
\sum_{j=1}^{n} c_{i j}=\sum_{k=0}^{n-1}\left[U_{k}^{(a, b)}\right]^{2}=\frac{M-\left[U_{n}^{(a, b)}\right]^{2}+B^{2}\left[U_{n-1}^{(a, b)}\right]^{2}}{(1-B)^{2}-A^{2}}+\frac{2\left(a^{2} B+a b A-b^{2}\right)\left[1-(-B)^{n}\right]}{(1+B)\left(A^{2}+4 B\right)}
$$

for any $i=1,2, \cdots, n$, where $M=a^{2}-(a A-b)^{2}-\frac{2(1+B)\left(a^{2} B+a b A-b^{2}\right)\left[1-(-B)^{n}\right]}{A^{2}+4 B}$. Hence

$$
\left\|\mathcal{A}_{U^{2}}\right\|_{2}=\|C\|_{2}=\frac{M-\left[U_{n}^{(a, b)}\right]^{2}+B^{2}\left[U_{n-1}^{(a, b)}\right]^{2}}{(1-B)^{2}-A^{2}}+\frac{2\left(a^{2} B+a b A-b^{2}\right)\left[1-(-B)^{n}\right]}{(1+B)\left(A^{2}+4 B\right)} .
$$

Thus the proof is completed.

In the particular case $A=B=1, a=0$ and $b=1$ from Theorem 2, we get the spectral norm for $g$-circulant matrix with the first row $\left(F_{0}^{2}, F_{1}^{2}, \cdots, F_{n-1}^{2}\right)$, which is the known result in [11].

Corollary 1 Let $\mathcal{A}_{F^{2}}$ be as (1.6), with $a_{i}=F_{i}^{2}(i=0,1, \cdots, n-1)$ in the first row of $\mathcal{A}_{F^{2}}$. If $(n, g)=1$, then we have

$$
\left\|\mathcal{A}_{F^{2}}\right\|_{2}=F_{n} F_{n-1} .
$$

Proof: We select $A=B=1, a=0$ and $b=1$ in Theorem 2, then the following is valid

$$
\left\|\mathcal{A}_{F^{2}}\right\|_{2}=F_{n}^{2}-F_{n-1}^{2}+(-1)^{n}
$$

Thus, the proof is completed from the following identity

$$
F_{n} F_{n-1}-\left(F_{n}^{2}-F_{n-1}^{2}\right)=F_{n+1} F_{n-1}-F_{n}^{2}=(-1)^{n} .
$$

In the case $A=B=1, a=2$ and $b=1$ from Theorem 2, we obtain the following result in [11].

Corollary 2 Let $\mathcal{A}_{L^{2}}$ be as (1.6), with $a_{i}=L_{i}^{2}(i=0,1, \cdots, n-1)$ in the first row of $\mathcal{A}_{L^{2}}$. If $(n, g)=1$, then we have the following identity

$$
\left\|\mathcal{A}_{L^{2}}\right\|_{2}=L_{n} L_{n-1}+2 .
$$

Proof: When $A=B=1, a=2$ and $b=1$ in Theorem 2, then we have

$$
\left\|\mathcal{A}_{L^{2}}\right\|_{2}=L_{n}^{2}-L_{n-1}^{2}-5(-1)^{n}+2 .
$$

On the other hand, applying identities $F_{n}+L_{n}=2 F_{n+1}$ and $F_{n+1} F_{n-1}-F_{n}^{2}=(-1)^{n}$, then we have

$$
\begin{aligned}
L_{n}^{2}-L_{n-1}^{2} & =\left(2 F_{n+1}-F_{n}\right)^{2}-\left(2 F_{n}-F_{n-1}\right)^{2} \\
& =4\left(F_{n+1}^{2}-F_{n+1} F_{n}-F_{n}^{2}\right)+\left(F_{n}^{2}-F_{n} F_{n-1}-F_{n-1}^{2}\right)+5 F_{n} F_{n-1} \\
& =3(-1)^{n}+5 F_{n} F_{n-1}
\end{aligned}
$$

hence, the following is valid

$$
\begin{aligned}
L_{n}^{2}-L_{n-1}^{2}-L_{n} L_{n-1} & =3(-1)^{n}+5 F_{n} F_{n-1}-\left(2 F_{n+1}-F_{n}\right)\left(2 F_{n}-F_{n-1}\right) \\
& =3(-1)^{n}+4\left(F_{n} F_{n-1}-F_{n+1} F_{n}\right)+2 F_{n+1} F_{n-1}+2 F_{n}^{2} \\
& =3(-1)^{n}+2\left(F_{n+1} F_{n-1}-F_{n}^{2}\right) \\
& =5(-1)^{n} .
\end{aligned}
$$

Thus the proof is completed.

\section{Numerical tests}

In this section, we list the results for Fibonacci and Lucas numbers in Table 1. Employing the formulas in above corollaries, the numerical results demonstrate that the explicit identities of spectral norms of $g$-circulant matrices hold exactly. 
Table 1. Numerical results of $a_{i}=F_{i}^{2}, L_{i}^{2}$

\begin{tabular}{|c|c|c|c|c|c|c|c|c|c|c|}
\hline$n$ & 7 & & & & & 9 & & & & \\
\hline$g$ & 2 & 3 & 4 & 5 & 6 & 2 & 4 & 5 & 7 & 8 \\
\hline$\left\|\mathcal{A}_{F^{2}}\right\|_{2}$ & 104 & 104 & 104 & 104 & 104 & 714 & 714 & 714 & 714 & 714 \\
\hline$\left\|\mathcal{A}_{L^{2}}\right\|_{2}$ & 524 & 524 & 524 & 524 & 524 & 3574 & 3574 & 3574 & 3574 & 3574 \\
\hline$F_{n} F_{n-1}$ & 104 & 104 & 104 & 104 & 104 & 714 & 714 & 714 & 714 & 714 \\
\hline$L_{n} L_{n-1}+2$ & 524 & 524 & 524 & 524 & 524 & 3574 & 3574 & 3574 & 3574 & 3574 \\
\hline
\end{tabular}

\section{Conclusion}

In this paper we introduce the notion of the classical Horadam numbers $U_{i}^{(a, b)}$, then give upper and lower bounds for the spectral norm of $g$-circulant matrix, whose the first row entries are $\left(U_{0}^{(a, b)}, U_{1}^{(a, b)}, \cdots, U_{n-1}^{(a, b)}\right)$. In addition, we also establish an explicit formula of the spectral norm for $g$-circulant matrix with the first row $\left(\left[U_{0}^{(a, b)}\right]^{2},\left[U_{1}^{(a, b)}\right]^{2}\right.$, $\left.\cdots,\left[U_{n-1}^{(a, b)}\right]^{2}\right)$. In two particular cases $A=B=1, a=0, b=1$ and $A=B=1, a=$ $2, b=1$, we obtain the known results from [11].

\section{Acknowledgement}

The authors thank the referees for their helpful suggestions concerning the presentation of this paper.

\section{References}

[1] S. Falcón, Á. Plaza, The $k$-Fibonacci sequence and the Pascal 2-triangle, Chaos, Solitons Fract. 33(2007) 38-49.

[2] S. Falcón, Á. Plaza, On $k$-Fibonacci numbers of arithmetic indexes, Appl. Math. Comput. 208(2009) 180-185.

[3] K. Uslu, N. Taskara, H. Kose, The generalized $k$-Fibonacci and $k$-Lucas numbers, Ars Combinatoria 99(2011) 25-32.

[4] P. Stanimirović, J. Nikolov, I. Stanimirović, A generalization of Fibonacci and Lucas matrices, Discrete Appl. Math. 156(2008) 2606-2619.

[5] M. Akbulak, D. Bozkurt, On the norms of Toeplitz matrices involving Fibonacci and Lucas numbers, Hacet. J. Math. Stat. 37(2008) 89-95.

[6] S.-Q. Shen, On the norms of Toeplitz matrices involving $k$-Fibonacci and $k$-Lucas numbers, Int. J. Contemp. Math. Science 7(2012) 363-368.

[7] S. Solak, On the norms of circulant matrices with the Fibonacci and Lucas numbers, Appl. Math. Comput. 160(2005) 125-132.

[8] S. Solak, Erratum to "On the norms of circulant matrices with the Fibonacci and Lucas numbers" [Appl. Math. Comput. 160(2005) 125-132], Appl. Math. Comput. 190(2007) 18551856.

[9] A. İpek, On the spectral norms of circulant matrices with classical Fibonacci and Lucas numbers entries, Appl. Math. Comput. 217(2011) 6011-6012.

[10] A. Bose, S. Guha, R.S. Hazra, K. Saha, Circulant type matrices with heavy tailed entries, Stat. Probab. Lett. 81(2011) 1706-1716.

[11] J. Zhou, Z. Jiang, The spectral norms of $g$-circulant matrices with classical Fibonacci and Lucas numbers entries, Appl. Math. Comput. 233(2014) 582-587.

[12] S.-Q. Shen, J.-M. Cen, Y. Hao, On the determinants and inverses of circulant matrices with Fibonacci and Lucas numbers, Appl. Math. Comput. 217(2011) 9790-9797.

[13] Y. Yazlik, N. Taskara, On the inverse of circulant matrix via generalized $k$-Horadam numbers, Appl. Math. Comput. 223(2013) 191-196. 
[14] S.-Q. Shen, J.-J. He, Moore-Penrose inverse of generalized Fibonacci matrix and its applications, Int. J. Comput. Math. 93(2016) 1756-1770.

[15] W.T. Stallings, T.L. Boullion, The pseudoinverse of an $r$-circulant matrix, Proc. AMS 34(1972) 385-388.

[16] R.A. Horn, C.R. Johnson, Topics in Matrix Analysis, Cambridge University Press, Cambridge, 1991. 